\title{
La depuración étnica en el derecho internacional: entre el derecho penal internacional y la responsabilidad de proteger
}

Ethnic Cleansing in International Law: Between International Criminal Law and the Responsibility to Protect

A depuração étnica no direito internacional: entre o direito penal internacional e a responsabilidade de proteger

Luciano Pezzano*

Fecha de recepción: 20 de marzo de 2020

Fecha de aprobación: 15 de junio de 2020 Doi: https://doi.org/10.12804/revistas.urosario.edu.co/anidip/a.9897

Para citar este artículo: Pezzano, L. (2020). La depuración étnica en el derecho internacional: entre el derecho penal internacional y la responsabilidad de proteger. ANIDIP 8, 1-27. https://doi.orģ/10.12804/ revistas.urosario.edu.co/anidip/a.9897

\section{Resumen}

La inclusión de la depuración étnica, un concepto que carece de significado jurídico propio en el derecho internacional, dentro del ámbito material de la responsabilidad de proteger, junto a crímenes bien tipificados en el derecho penal internacional, ha dado lugar a ciertas dificultades conceptuales. Este ensayo busca explorar, a través de la caracterización de la depuración étnica en el derecho internacional $y$, en particular, de sus posibles configuraciones como crímenes según el derecho penal internacional, los problemas que esa inclusión a primera vista anómala puede plantear en la interpretación del alcance material de esta. Con tal fin, se aborda el nacimiento y evolución del concepto de la depuración étnica en las relaciones internacionales y en su consideración por los órganos de las Naciones Unidas; se

Aboģado (UCES); maģíster en Relaciones Internacionales (CEA-UNC); doctor en Derecho y Ciencias Sociales (Unc); becario posdoctoral cIJs-unc/Conicet. Profesor titular de Derechos Humanos desde la Perspectiva Internacional (UCES); profesor asociado de Derecho Internacional Público y de la Integración (UCEs); profesor ayudante 'A' de Derecho Internacional Público (Facultad de Derecho, unc). 
lo analiza luego a la luz del derecho penal internacional, para determinar si puede constituir uno o más de los crímenes internacionales reconocidos, teniendo en cuenta la jurisprudencia internacional y las normas aplicables; finalmente, se estudia la inclusión de la depuración étnica en el ámbito de la responsabilidad de proteger y los problemas que plantea, para elaborar una posible alternativa de solución a partir de los resultados del análisis.

Palabras clave: depuración étnica; genocidio; crímenes de lesa humanidad; crímenes de guerra; responsabilidad de proteger.

\section{Abstract}

The inclusion of ethnic cleansing, a concept that has no legal significance of its own in international law, within the material scope of the responsibility to protect (R2P), together with crimes well-defined in international criminal law, has led to certain conceptual difficulties. This article presents the exploration, through the characterization of ethnic cleansing in international law and, in particular, its possible configurations as crimes under international criminal law, of the issues that such anomalous inclusion may pose in the interpretation of the material scope of the R2P. To this end, the origins and evolution of the concept of ethnic cleansing in international relations and its consideration by the United Nations organs are addressed. Then it presents the analysis in the light of international criminal law to determine whether it may constitute one or more of the recognized international crimes, taking into account international jurisprudence and applicable rules. Finally, the inclusion of ethnic cleansing in the context of the $\mathrm{R} 2 \mathrm{P}$ and the issues it poses are explored in order to develop a possible solution based on the results of the analysis.

Keywords: Ethnic cleansing; genocide; crimes against humanity; war crimes; responsibility to protect.

\section{Resumo}

A incluso da depuração étnica, um conceito que carece de significado jurídico próprio no direito internacional, dentro do âmbito material da responsabilidade de proteger, junto a crimes bem tipificados no direito penal internacional, tem dado lugar a certas dificuldades conceituais. Este ensaio busca explorar, através da caracterização da depuração étnica no direito internacional e, em particular, de suas possíveis configurações como crimes segundo o direito penal internacional, os problemas que essa inclusão à primeira vista anómala pode apresentar na interpretação do alcance material dela. Com este fim, se aborda o nascimento e evolução do conceito da depuração étnica nas relações internacionais e em sua consideração pelos órgãos das 
Nações Unidas; analisa-o logo à luz do direito penal internacional, para determinar se pode constituir um ou mais dos crimes internacionais reconhecidos, tendo em consideração a jurisprudência internacional e as normas aplicáveis; finalmente, estuda-se a inclusão da depuração étnica no âmbito da responsabilidade de proteger e os problemas que apresenta, para elaborar uma possível alternativa de solução a partir dos resultados da análise.

Palavras-chave: depuração étnica; genocídio; crimes de lesa humanidade; crimes de guerra; responsabilidade de proteger.

\section{Introducción}

Pese a ser una de las más odiosas violaciones a los derechos humanos, la 'depuración étnica' (DE) no constituye, como tal, una categoría jurídica autónoma en el derecho internacional, ni posee una definición legalmente aceptada. Aunque no es en sí misma un crimen internacional, puede, de acuerdo con las modalidades y contexto de su ejecución, constituir genocidio, o uno o varios crímenes de guerra o de lesa humanidad.

No obstante esta falta de autonomía jurídica, fue incluida dentro del alcance material de la responsabilidad de proteger (RDP), tal cual fue receptada por las $\mathrm{Na}$ ciones Unidas (NU) en el Documento final de la Cumbre Mundial 2005 (DF), junto con las tres categorías de crímenes internacionales antes referidas. Esta inclusión ha dado lugar a ciertas dificultades conceptuales al momento de referirse al ámbito material de la RDP: ¿significa que la DE tiene en el contexto de la RDP un significado jurídico propio? O, por el contrario, al carecer de sentido jurídico autónomo, ¿se trata de una anomalía en el DF? En todo caso, ¿por qué se produjo?

El propósito de este ensayo es, entonces, explorar, a través de la caracterización de la DE en el derecho internacional y, en particular, de sus posibles configuraciones como crímenes según el derecho penal internacional, los problemas que su inclusión junto a estos en el ámbito de la RDP puede plantear en la interpretación del alcance material de esta, por tratarse de un concepto que carece de un significado jurídico preciso que acompaña a categorías bien definidas del derecho penal internacional.

Cabe recordar que, desde la perspectiva de la RDP, los autores no se han detenido particularmente en esta cuestión, más allá de aclarar que la DE no es un crimen en sí misma conforme con el derecho internacional. Sin embargo, no reflexionan sobre qué sentido tiene su inclusión dentro del ámbito material de la RDP ni, mucho menos, por qué es problemática. Este estudio pretende, entonces, abordar estas cuestiones desatendidas por la mayoría de la doctrina. 
Con tal fin, comenzaremos abordando la cuestión del nacimiento y evolución del concepto de la DE en las relaciones internacionales, haciendo especial hincapié en su consideración por los órganos de las NU, para luego analizarlo a la luz del derecho penal internacional, en aras de determinar si puede constituir uno o más de los crímenes internacionales reconocidos, teniendo en cuenta la jurisprudencia internacional y las normas aplicables; estudiaremos la inclusión de la DE en el ámbito material de la RDP y los problemas que plantea, teniendo presente el resultado de nuestro anterior análisis, lo que nos lleva a elaborar una posible alternativa de solución a la cuestión problemática.

\section{La depuración étnica}

\subsection{Etimología y origen histórico}

La DE es una práctica de larga data en los conflictos armados; no obstante, tomó dramática actualidad con ese nombre durante las guerras en la ex-Yugoslavia en los años noventa, cuando fue rápidamente adoptada por los medios de comunicación en sus informes y notas sobre la situación en diferentes áreas de Bosnia y Croacia.

Su etimología puede encontrarse en el serbocroata etničko čišćenje, que literalmente significa "limpieza étnica" (Andreopoulos, 2004). Sin embargo, como señala Petrovic (1994), su origen histórico es difícil de establecer: hay informes de medios de comunicación que discutieron el establecimiento de "territorios étnicamente limpios" en Kosovo después de 1981, pero el término se difundió masivamente y adquirió su significado actual durante la guerra en Bosnia y Herzegovina (p. 343).

La expresión se tradujo literalmente al inglés como "ethnic cleansing" $\mathrm{y}$ al francés como "nettoyage ethnique". En castellano, los documentos oficiales de las Nu emplean indistintamente "limpieza étnica" y "depuración étnica" (sin haberse nunca explicitado por qué la opción por uno u otro término), aunque en los documentos relativos a la RDP siempre se ha utilizado "depuración étnica", que es la que preferiremos aquí.

Aunque de fuente incierta, la expresión puede tener origen en la jerga castrense respecto de 'limpiar' determinada área de operaciones, ${ }^{2}$ dado el rol preponderante

1 En inglés, cleansing, del verbo cleanse, deriva de clean, 'limpiar', pero en el sentido más específico de deshacerse de impurezas (Merriam-Webster Dictionary, definition of "cleanse", s. f.), es decir, dejar algo completamente limpio o puro (Cambridge Dictionary, definition of "cleanse", s. f.).

2 Al respecto, podemos recordar que la Sala de Apelaciones del tpiY en el caso Kordić debió enfrentarse a una discusión lingüística, dado que la Sala de Primera Instancia había valorado como decisivo el testimonio de un testigo que afirmó haber participado en una "operación de limpieza" (cleansinǵ operation), que había sido entendida como "limpieza étnica", y la defensa sostenía que debía entenderse como una operación de limpieza (mopping up), común en el lenguaje militar en casos de combate urbano (que se refiere a las operaciones posteriores al combate en las que se busca neutralizar las fuerzas y materiales del enemigo que pudieron haber permanecido; de allí que se trate de 'limpiar' la zona). La Sala rechazó la arģumentación de la defensa: "The Appeals Chamber notes that in this context 
que jugaron oficiales militares del ex Ejército Popular Yugoslavo en los eventos en Bosnia. La expresión "limpiar el territorio" se dirige contra los enemigos, y es utilizada principalmente en la fase final del combate en orden de adquirir control total sobre el territorio ocupado. Es probable que el adjetivo 'étnico' se haya agregado al término militar porque los 'enemigos' eran considerados tales por su pertenencia a otras comunidades étnicas (Petrovic, 1994, p. 343).

\subsection{La depuración étnica en los órganos de las Naciones Unidas}

Prácticamente desde el comienzo de la consideración por parte de los órganos de las NU de la situación en la ex-Yugoslavia, la DE formó parte de la discusión.

En julio de 1992, el Consejo de Seguridad (cs) empleó por primera vez el término en la Resolución 771 (1992), en la que condenó “enérgicamente las violaciones del derecho internacional humanitario, especialmente la que entraña la práctica de 'limpieza étnica". Así, el cs la calificó como una "práctica" que constituye una violación del derecho internacional humanitario.

En agosto de ese año, la Asamblea General (AG), en su Resolución 46/242, sobre la situación en Bosnia y Herzegovina, "condenando enérgicamente la práctica odiosa de 'depuración étnica', que constituye una grave y seria violación del derecho internacional humanitario", condenó "las violaciones masivas de los derechos humanos y del derecho internacional humanitario, especialmente la práctica odiosa de 'depuración étnica", exigió "que se ponga fin de inmediato a esta práctica y que se adopten otras medidas, con carácter urgente, para detener el desplazamiento masivo y forzoso de la población”, e instó “a todos los Estados y organizaciones internacionales a que no reconozcan las consecuencias [...] de la práctica odiosa de 'depuración étnica”. Además de compartir la caracterización del cs, la AG la calificó como una "práctica odiosa" y la vinculó al desplazamiento masivo y forzoso de la población.

Unos días antes, la Comisión de Derechos Humanos (CDH), en su primer período extraordinario de sesiones, había aprobado la Resolución 1992/S-1/1, en la que, "expresando su particular rechazo del concepto y la práctica de la 'limpieza étnica' [...], que como mínimo entraña las deportaciones y los traslados o expulsiones forzados y masivos de personas de sus casas en violación flagrante de sus derechos

the expression 'cleansing operation' is ambiguous since it could be understood as synonymous with 'mopping up operation' in the military sense or with unlawful 'ethnic cleansing'. The Appeals Chamber notes further that, in the presence of a compound question such as the one containing the Prosecution's description of the attacks in which Witness As participated as an 'ethnic cleansing campaign', a trier of fact must be particularly cautious when interpreting the answer given by the witness. However, read in context, the answer 'yes' given by Witness As applied to all aspects of this question, including the ethnic connotation, supported by his answer that looting was also exclusively directed against Muslims. Additionally, there was nothing to 'mop up' in a military sense" (Kordić, párr. 403). El caso es ilustrativo de lo compleja que puede ser la cuestión semántica en torno a la DE. 
humanos, con el objeto de disgregar o destruir los grupos nacionales, étnicos, raciales o religiosos", condenó "de modo absoluto el concepto y la práctica de la 'limpieza étnica". De modo interesante, la Comisión aporta el primer intento de definición de DE para un órgano de las NU.

En la Resolución 787 (1992), el cs reafirmó que "toda práctica de 'limpieza étnica' es ilícita e inaceptable", reiteró su condena a "la práctica de la "limpieza étnica"3 y reafirmó "que se considerarán responsables individualmente de esos actos a aquellos que los cometan o que ordenen su comisión", ${ }^{3}$ lo que reiteró en su Resolución 819 (1993), en la que condenó la "abominable campaña general de 'depuración étnica". El cs dio un paso más al reconocer la responsabilidad individual de los autores o instigadores de la DE, indicando así su naturaleza de crimen (Pégorier, 2013, p. 18).

El relator especial de la CDH sobre la situación de derechos humanos en la ex-Yugoslavia sostuvo que "el principal objetivo del conflicto militar en Bosnia y Herzegovina es el establecimiento de regiones étnicamente homogéneas. La limpieza étnica no parece ser la consecuencia de la guerra sino más bien su objetivo. Este objetivo, en gran medida, ya ha sido alcanzado por medio de matanzas, palizas, violaciones, destrucción de casas y amenazas" (NU, 1992, p. 4).

En el mismo informe, definió la DE como

la eliminación de miembros de grupos étnicos por otro grupo étnico que ejerce control sobre un territorio determinado. Para alcanzar este fin se utiliza una amplia variedad de métodos, incluidas las amenazas, el hostigamiento y la intimidación; los disparos o el empleo de explosivos en hogares, tiendas y sitios donde se realizan operaciones comerciales, la destrucción de los lugares de culto y las instituciones culturales; la transferencia o reubicación por la fuerza de las poblaciones; ejecuciones sumarias; atrocidades cometidas con el propósito determinado de infundir terror entre la población, como la tortura, la violación y la mutilación de los cadáveres, y el bombardeo de centros de población civil (NU, 1992, p. 5).

En la Resolución 47/121, la AG, tomando nota del informe, se mostró seriamente preocupada por una pauta constante de violaciones graves y sistemáticas de los de-

3 También hay expresiones de alarma por la práctica de la 'limpieza étnica' en el preámbulo de las resoluciones 779 (1992), 780 (1992), 808 (1993) y 827 (1993), y condenas en las resoluciones 820 (1993), 824 (1993), 836 (1993) y 859 (1993).

4 La AG, en su Resolución 47/80, reiteró su convicción de que quienes cometen u ordenan que se cometan actos de "depuración étnica" son responsables a título individual de esos actos y deben ser procesados, lo que también se sostuvo en la Declaración y Programa de Acción de Viena (Conferencia Mundial de Derechos Humanos, 1993, p. 18). 
rechos humanos, una creciente población de refugiados resultante de la expulsión masiva de civiles, "en aplicación de la abominable política de 'limpieza étnica', que constituye una forma de genocidio". Aquí, la AG no solamente la calificó como una "abominable política", es decir, no solamente una "práctica" (sobre lo que volveremos), sino que directamente la consideró como una forma de genocidio.

El cs pidió al Secretario General (sG) que estableciera una Comisión de Expertos para que informara sobre las violaciones del derecho internacional humanitario en la ex-Yugoslavia. En su informe preliminar, la Comisión sostuvo: "La expresión 'depuración étnica' es relativamente nueva. En el contexto de los conflictos en la ex Yugoslavia la práctica de 'depuración étnica' consiste en dar homogeneidad étnica a una zona utilizando la fuerza o la intimidación para expulsar a personas o a determinados grupos de dicha zona. La 'depuración étnica' contraviene el derecho internacional" (cs, 1993b, p. 15).

Además de dar su definición - la que como veremos será una de las más utilizadas-, la Comisión también describió cómo se llevó a cabo "mediante asesinatos, torturas, detenciones y encarcelamientos arbitrarios, ejecuciones sin beneficio de proceso judicial, violaciones y agresiones sexuales, confinación de poblaciones civiles en zonas de gueto, expulsiones por la fuerza, desplazamientos y deportación de poblaciones civiles, ataques militares deliberados o amenazas de ataque contra civiles y zonas civiles, y destrucción inexcusable de bienes" (cs, 1993b, p. 15).

La Comisión también hizo una valoración jurídica: "Estas prácticas constituyen crímenes de lesa humanidad y pueden asimilarse a crímenes de guerra concretos. Esos actos también podrían considerarse comprendidos en el marco de la Convención para la Prevención y la Sanción del Delito de Genocidio" (cs, 1993b, p. 15).

En su informe, la Comisión consideró que la creación de un tribunal internacional ad hoc estaría de acuerdo con la orientación de sus trabajos. El cs decidió establecer dicho tribunal penal internacional en su Resolución 808 (1993), encargando al sG la presentación de un informe al respecto. En dicho informe el sG no solo incluyó la DE dentro de los crímenes de lesa humanidad, sino que también la vinculó a formas de violencia sexual que se emplearon durante los conflictos en la ex-Yugoslavia (cs, 1993a, pp. 13-14).

En su informe final, la Comisión de Expertos reiteró los términos de su informe preliminar y afirmó "que la 'depuración étnica’ es una política deliberada concebida por un grupo étnico o religioso para desplazar por la violencia y el terror a la población civil de otro grupo étnico o religioso de determinadas zonas geográficas" (CS, 1994, p. 34). La Comisión describió los medios y la forma en que la DE fue llevada a cabo, y concluyó que existía una política en ese sentido y que se aplicó de manera 
sistemática (cs, 1994, pp. 34-36). Igualmente, sostuvo que "había una política dominante en favor de utilizar la violación como método de 'depuración étnica” (cs, 1994, p. 59), pero no realizó ninguna consideración jurídica diferente a la que había hecho en su informe preliminar.

Por su parte, el Tribunal Penal Internacional para la ex-Yugoslavia (TPIY) menciona en numerosas oportunidades a la DE en su jurisprudencia, pero rara vez la define o aporta sus elementos característicos. De hecho, en el caso Krajišnik, se sostuvo: "With regard to the term 'ethnic cleansing', which was sometimes used by witnesses or in reports, the Chamber wishes to note that it has not treated the term as legally significant” (Krajišnik, párr. 293). Así que, por lo general, se usa para describir hechos o conductas, o incluso un panorama histórico, como se hizo en Tadić (párr. 84). En otras sentencias, el TPIY ha efectuado algunos aportes para caracterizar la DE dentro de los crímenes de su competencia, como lo veremos más adelante.

Finalmente, la Corte Internacional de Justicia (CIJ), en su sentencia sobre el fondo en el caso Genocidio, dijo: “The term 'ethnic cleansing' has frequently been employed to refer to the events in Bosnia and Herzegovina which are the subject of this case" (Genocidio, párr. 190). La Corte se refiere a las resoluciones de la AG y el cs, así como al informe de la Comisión de Expertos, y considera: "It will be convenient at this point to consider what legal significance the expression may have" (Genocidio, párr. 190), para lo cual adoptó la definición del informe preliminar de la Comisión de Expertos, y continuó con su análisis jurídico a la luz de las disposiciones de la Convención para la Prevención y Sanción del Delito de Genocidio (la Convención de 1948), según veremos más adelante.

\subsection{Hacia una definición de la depuración étnica}

Este repaso por las diferentes opiniones y acciones de los órganos de las NU nos permite abordar la cuestión de la definición y caracteres de la DE en la práctica internacional.

Comenzaremos, por supuesto, por la definición de la Comisión de Expertos, que, al haber sido empleada por la CIJ, tiene un gran peso en la discusión. Conforme con la Comisión, la DE es una práctica que consiste en dar homogeneidad étnica a una zona utilizando la fuerza o la intimidación para expulsar a personas o a determinados grupos de dicha zona.

La definición es útil por su sencillez, y reúne varios de los elementos que la doctrina ha identificado como caracterizadores de la DE. Bulutgil (2016) identifica tres criterios como particularmente importantes: los autores de la violencia, que son los líderes de un Estado - en cuanto son los capaces de formular y llevar a cabo una 
política de $\mathrm{DE}$-; el blanco de la violencia, que es un grupo étnico y no individuos seleccionados por otros criterios; y los métodos que los autores emplean para llevar a cabo la violencia - que implican que las poblaciones son desplazadas involuntaria y permanentemente-. Estos criterios le permiten definir la DE como cualquier evento en el que una organización que tiene la capacidad de usar la coerción en un territorio dado deporta permanentemente y/o mata una parte sustancial de un grupo étnico que vive dentro de su territorio (Bulutgil, 2016, pp. 4-7).

Petrovic (1994) llama la atención sobre la forma de caracterizar la DE, y que, como hemos visto, oscila entre su consideración como una "práctica" o una "política". Si se caracteriza como una "práctica”, se está apuntando a la descripción de las acciones que tienen lugar en el área donde se está llevando a cabo; esa fue la primera aproximación de los órganos de las NU, tanto el Cs como la AG y la CDH, así como la Comisión de Expertos en su informe preliminar, la calificaron de esa manera. Pero si, en cambio, se la describe como una "política", se apunta a la existencia de un plan ${ }^{5}$ y de una intención detrás de esas acciones. Fue el relator especial de la $\mathrm{CDH}$ quien primero la caracterizó como una política desde su primer informe (CDH, 1992), enfoque luego seguido por la AG y por la Comisión de Expertos en su informe definitivo, al igual que por el Comité para la Eliminación de la Discriminación Racial, que se refiere a la existencia de una "política sistemática" de DE (CERD, 1996, p. 41).

En función de ello, nos permitimos, a los fines de este ensayo, reformular los criterios identificados por Bulutgil de la siguiente manera: 1) la existencia de una práctica generalizada de conformidad con la política formulada por un Estado u organización; 2) la finalidad discriminatoria dirigida en contra de uno o más grupos étnicos con miras a su eliminación (mediante la destrucción o la expulsión) de determinado territorio; y 3 ) el empleo de medios violentos que implican diferentes formas de uso de la fuerza y la coacción, contrarios al derecho internacional, para lograr tal finalidad.

La noción de DE no está exenta de críticas. Dos son las principales que se han dirigido contra el concepto. La primera es que es un concepto sin contenido ni valor jurídico que parece concebido para ser un eufemismo de 'genocidio' y así eludir las obligaciones internacionales que se derivan de la Convención de 1948 (Blum, Stanton, Sagi \& Richter, 2008). Esta crítica puede tener alguna repercusión al momento de analizar la DE en el contexto de la RDP, por lo que volveremos sobre ella.

5 De hecho, la existencia de una 'política' es uno de los elementos que caracterizan el "ataque contra una población civil” en la definición de los crímenes de lesa humanidad de acuerdo con el artículo 7.2.a) del ER. 
La segunda crítica refiere a la connotación peyorativa del término (Pégorier, 2013, p. 2), ya que fue creado por los propios perpetradores - lo que explica que en los documentos de las NU se haya empleado principalmente entre comillas-, y su reiteración puede implicar, en cierta forma, condonar la utilización de un eufemismo ('limpieza') que encubre una atroz violación de derechos humanos. Aunque compartimos esta apreciación, lo cierto es que su uso se ha generalizado en la práctica y en los documentos relativos a la RDP ya ni siquiera figura entre comillas.

\section{La depuración étnica en el derecho penal internacional}

Al no estar tipificada como tal por el derecho internacional, es necesario contrastar la DE con los crímenes de derecho penal internacional a los fines de determinar si puede ser identificada con alguno de ellos.

\subsection{La depuración étnica como genocidio}

En su Resolución 47/121, la AG consideró que la DE constituye una "forma de genocidio", aunque no elaboró más al respecto. Por su parte, el TPIY en el caso Krstić sostuvo que existían "obvias similitudes" entre una política genocida y la política comúnmente conocida como "depuración étnica" (Krstić, párr. 562). Esto es así por la finalidad discriminatoria que inspira a ambas figuras, pero no es suficiente para equipararlas, ni para considerar, como la $\mathrm{AG}$, que la $\mathrm{DE}$ como tal es una forma de genocidio.

La relación quedó establecida con mayor claridad (Schabas, 2007, p. 109) en la sentencia de la CIJ en la causa Genocidio. Allí, la Corte recordó que la expresión “depuración étnica” no aparece en la Convención de 1948 y que, de hecho, una propuesta durante la redacción de la Convención para incluir en la definición las "medidas con la intención de obligar a miembros de un grupo a abandonar sus hogares en orden a escapar de la amenaza de ulteriores malos tratos" no fue aceptada (Genocidio, párr. 190). En efecto, la Corte se refirió a una enmienda propuesta por Siria (AG, 1948a) que se rechazó principalmente porque la mayoría de las delegaciones en la Sexta Comisión de la AG entendía que, si bien se trataba de una conducta de cierta gravedad, el genocidio implicaba la destrucción de un grupo humano, y que aceptar la propuesta habría resultado en una ampliación del concepto de genocidio (AG, 1948b). ${ }^{6}$ 
La CIJ argumentó que la DE solo puede constituir una forma de genocidio dentro del significado de la Convención si corresponde o cae dentro de una de las categorías de actos prohibidos por el artículo II: ${ }^{7}$

Neither the intent, as a matter of policy, to render an area "ethnically homogeneous", nor the operations that may be carried out to implement such policy, can as such be designated as genocide: the intent that characterizes genocide is "to destroy, in whole or in part" a particular group, and deportation or displacement of the members of a group, even if effected by force, is not necessarily equivalent to destruction of that group, nor is such destruction an automatic consequence of the displacement (Genocidio, párr. 190).

Esto no significa, aclaró la Corte, que los actos descritos como DE nunca puedan constituir genocidio, si pueden caracterizarse como, por ejemplo, "sometimiento intencional del grupo a condiciones de existencia que hayan de acarrear su destrucción física, total o parcial", contrario al artículo II, párrafo c), de la Convención, siempre que tal acción se lleve a cabo con la intención específica (dolus specialis) necesaria, es decir, con miras a la destrucción de un grupo, diferente de la intención de removerlo de una región (Genocidio, párr. 190).

No es casual que la CIJ haya escogido el supuesto del inciso c) del artículo II como ejemplo de acto genocida en el que podría estar incluida la DE. En su Proyecto de Código de Crímenes contra la Paz y Seguridad de la Humanidad, la Comisión de Derecho Internacional (CDI) incluyó el genocidio en su artículo 17, en el que siguió textualmente el artículo II de la Convención. En el comentario, al referirse al apartado c), la CDI señala que se sugirió incluir en este la deportación, pero que estimó "que el apartado comprendía la deportación en los casos en los que con ésta se pretende destruir el grupo, total o parcialmente" (CDI, 2002, p. 50). También los Elementos de los Crímenes del Estatuto de Roma de la Corte Penal Internacional (ER) confirman esta interpretación. Así, el elemento 4 del crimen de genocidio mediante sometimiento intencional a condiciones de existencia que

$7 \quad$ El artículo II de la Convención de 1948 dispone: “En la presente Convención, se entiende por genocidio cualquiera de los actos mencionados a continuación, perpetrados con la intención de destruir, total o parcialmente, a un ģrupo nacional, étnico, racial o reliģioso, como tal: a) matanza de miembros del grupo; b) lesión grave a la integridad física o mental de los miembros del ģrupo; c) sometimiento intencional del grupo a condiciones de existencia que hayan de acarrear su destrucción física, total o parcial; d) medidas destinadas a impedir los nacimientos en el seno del grupo; e) traslado por fuerza de niños del grupo a otro grupo". El artículo 6ㅇ del ER reproduce textualmente la disposición.

8 Aunque la Corte no lo señale en apoyo de su afirmación - ya que su competencia estaba limitada a la interpretación y aplicación de la Convención-, los Elementos de los Crímenes del er confirman su opinión, al requerir, en cada caso, la intención del autor de destruir total o parcialmente un ǵrupo. 
hayan de acarrear su destrucción física incluye la siguiente nota: "La expresión 'condiciones de existencia' podrá incluir, entre otras cosas, el hecho de privar a esas personas de los recursos indispensables para la supervivencia, como alimentos o servicios médicos, o de expulsarlos sistemáticamente de sus hogares" (énfasis añadido). Ambas fuentes, aunque no mencionen expresamente la DE, sí admiten que su contenido puede coincidir con actos genocidas, como lo indicó la CIJ, siempre que se satisfaga el dolo específico.

La CIj también recurre a la jurisprudencia del TPIY, recordando lo que afirmó en el caso Krstić, pero igualmente lo que se sostuvo en el caso Stakić, respecto a que debe hacerse una clara distinción entre la destrucción física y la mera disolución de un grupo: "The expulsion of a group or part of a group does not in itself suffice for genocide" (Stakić, párr. 519).

Así, la cij concluye:

Whether a particular operation described as "ethnic cleansing" amounts to genocide depends on the presence or absence of acts listed in Article II of the Genocide Convention, and of the intent to destroy the group as such. In fact, in the context of the Convention, the term "ethnic cleansing" has no legal significance of its own. That said, it is clear that acts of "ethnic cleansing" may occur in parallel to acts prohibited by Article II of the Convention, and may be significant as indicative of the presence of a specific intent (dolus specialis) inspiring those acts (Genocidio, párr. 190).

La Corte aclara, entonces, que la DE no es en sí misma una forma de genocidio - negando así, aunque implícitamente, la afirmación de la AG-, y que para que constituya genocidio deben cometerse alguno de los actos enunciados en el artículo II de la Convención con el dolo específico de destrucción de un grupo nacional, racial, étnico o religioso (Pégorier, 2013, p. 70) —confirmando así la idea que subyació al rechazo de la enmienda siria durante la redacción de la Convención-, pero sí parece acordar con la opinión del TPIY en Krstić, en cuanto a que la DE sí puede usarse como indicio de la existencia de una intención genocida, idea que ya había sido adelantada por la doctrina (Quigley, 2006, p. 195).

De este modo, aunque no pueda trazarse una equivalencia lineal entre el genocidio y la $\mathrm{DE}$, la jurisprudencia nos permite afirmar que esta sí existirá en cuanto se satisfagan los requisitos del artículo II de la Convención de 1948 y del artículo $6^{\circ}$ del ER. Pero ¿qué sucede cuando la práctica de DE no cumple con esos requisitos? 


\subsection{La depuración étnica como crimen de lesa humanidad}

Comentando la sentencia de la CIJ en Genocidio, Schabas (2016) expresó que el debate acerca de si la DE estaba o no subsumida dentro de la definición de genocidio perdía su relevancia en el contexto de la CPI porque incluso si no satisficiera la definición, estaba indudablemente cubierta por el artículo $7^{\circ}$ del ER, relativo a los crímenes de lesa humanidad (p. 137).

En efecto, de acuerdo con el artículo 7.1 del ER, los crímenes de lesa humanidad requieren, para ser calificados como tales, ser cometidos "como parte de un ataque generalizado o sistemático contra una población civil”. El artículo 7.2.a) del ER define al "ataque contra una población civil" como "una línea de conducta que implique la comisión múltiple de actos mencionados en el párrafo 1 contra una población civil, de conformidad con la política de un Estado o de una organización de cometer esos actos o para promover esa política".

La DE, prácticamente por definición, satisface el elemento de contexto establecido por el ER: está dirigida contra una población civil y se corresponde con una política diseñada por un Estado o por una organización - en el caso de los Balcanes, los grupos paraestatales formados por los serbios de Bosnia-. En cuanto a los requisitos de ser un ataque "generalizado" o "sistemático", el TPIY ya se había pronunciado. Sobre el primero, sostuvo en Nikolić: "The implementation of that discriminatory policy, commonly referred to as 'ethnic cleansing', over the region of Vlasenica alone seems to have been so widespread as to fall within the Tribunal's jurisdiction under Article 5" (Nikolić, párr. 27). Sobre el segundo, afirmó en Karadzić y Mladić que "a deliberate and systematic line of conduct called 'ethnic cleansing' has been substantiated" (Karadzić y Mladić, párr. 59).

Satisfecho el elemento de contexto, corresponde analizar cuál o cuáles de los actos enumerados en el artículo $7^{\circ}$ del ER se corresponden con la DE, aclarando desde ya que, como se dijo, los medios de su comisión son múltiples y podrían caer dentro de varios crímenes de lesa humanidad.

\subsubsection{Exterminio}

Contemplado en el artículo 7.1.a) del ER, el exterminio consiste, conforme con los Elementos de los Crímenes, en una matanza de miembros de una población civil, incluso mediante la imposición de condiciones de existencia destinadas deliberadamente a causar la destrucción de parte de una población. En la medida en que la DE puede llevarse a cabo mediante matanzas, como lo advirtieron en su momento los órganos de las NU respecto de la situación en los Balcanes, tales conductas quedarán incluidas bajo el crimen de lesa humanidad de exterminio. Aunque este 
no exige una finalidad discriminatoria, ello no es óbice para considerar incluidos actos de DE mientras los elementos materiales y mentales se satisfagan.

Sin embargo, de ello no debe derivarse una total asimilación entre la DE y el exterminio, toda vez que aquella puede llevarse a cabo mediante otros medios que no caerán dentro de este.

\subsubsection{Deportación o traslado forzoso de población}

La trágica experiencia de los Balcanes ha hecho que probablemente el crimen de lesa humanidad previsto en el artículo 7.1.d) del ER sea el más asociado a la idea de la DE, dado que, de acuerdo con los Elementos de los Crímenes, requiere para su configuración que el autor haya deportado, desplazado o trasladado por la fuerza (que no se limita a la fuerza física, sino que puede incluir la amenaza de la fuerza o la coacción, como la causada por el temor a la violencia, la intimidación, la detención, la opresión psicológica o el abuso de poder, contra esa o esas personas u otra o aprovechando un entorno de coacción), sin motivos autorizados por el derecho internacional y mediante la expulsión u otros actos de coacción, a una o más personas a otro Estado o lugar.

Como modalidad de DE, es la más frecuentemente descripta en los informes y resoluciones de los órganos de las NU. De hecho, en su Resolución 1997/29, la Subcomisión de Prevención de la Discriminación y Protección a las Minorías incluyó la "limpieza étnica" como una forma de desplazamiento forzoso de poblaciones.

\subsubsection{Crímenes sexuales}

En el artículo 7.1.g) del ER se tipifican diferentes conductas de violencia sexual como crímenes de lesa humanidad. Como vimos, ya la Comisión de Expertos había llamado la atención sobre este punto, señalando que en los conflictos de la ex-Yugoslavia las violaciones y otros abusos sexuales formaban parte de una campaña sistemática de DE. También es interesante destacar a este respecto la figura del crimen de embarazo forzado, ya que requiere para su configuración la "intención de modificar la composición étnica de una población”, intención presente en una política de DE.

\subsubsection{Persecución}

El artículo 7.1.h) del ER califica como crimen de lesa humanidad la "persecución de un grupo o colectividad con identidad propia fundada en motivos políticos, raciales, nacionales, étnicos, culturales, religiosos, de género [...], en conexión con cualquier acto mencionado en el presente párrafo o con cualquier crimen de la competencia de la Corte". 
El crimen de lesa humanidad de persecución es particularmente cercano a la idea de DE (Pégorier, 2013, p. 107). Por un lado, comparten la finalidad discriminatoria (aunque en aquel es más amplio que en esta) y reconoce a la vez la multiplicidad de medios de comisión, al establecer una conexión con cualquier crimen de competencia de la CPI. Así mismo, fue el crimen cuya conexión con la DE más se discutió explícitamente en la jurisprudencia del TPIY. Así, en Kupreškić, la Sala de Primera Instancia indicó:

If persecution was given a narrow interpretation, so as not to include the crimes found in the remaining sub-headings of Article 5, a lacuna would exist in the Statute of the Tribunal. There would be no means of conceptualising those crimes against humanity which are committed on discriminatory grounds, but which, for example, fall short of genocide, which requires a specific intent "to destroy, in whole or in part, a national, ethnical, racial, or religious group". An example of such a crime against humanity would be the so-called "ethnic cleansing", a notion which, although it is not a term of art, is particularly germane to the work of this Tribunal (Kupreškić, párr. 606).

De esta forma, el TPIY reconoció que, aunque la DE no es un concepto jurídico, sí la consideró dentro del crimen de persecución, precisamente por su finalidad discriminatoria.

En definitiva, aunque no pueda identificarse con un crimen de lesa humanidad en particular, los medios para llevar a cabo una campaña de DE pueden constituir varios de estos crímenes, y la propia política de DE provee el elemento de contexto necesario para la calificación de estas conductas.

\subsection{La depuración étnica como crimen de guerra}

Si podemos definir sucintamente a los crímenes de guerra como violaciones graves de las normas del derecho internacional humanitario (Ambos, 2011, p. 20), el hecho de que los órganos de las Nu hayan considerado casi desde el comienzo que la política de DE constituye una violación de este tipo la acerca a esta noción.

La pregunta que de inmediato surge es a cuál de los múltiples crímenes de guerra tipificados en el extenso artículo $8^{\circ}$ del ER podemos asimilar la DE. La respuesta, al igual que sucede con los crímenes de lesa humanidad, lleva a identificar múltiples crímenes de guerra que pueden formar parte de una campaña de DE. Esto puede incluir, entre otros, crímenes sexuales, tipificados en el artículo 8.2.b.xxii) del ER para los conflictos armados internacionales y en el artículo 8.2.e.vi) para los conflictos 
sin carácter internacional, y en particular, por su finalidad cercana a la DE, el crimen de embarazo forzado (Cottier \& Mzee, 2016, p. 499), o el crimen de guerra más genérico de dirigir ataques contra una población civil - artículo 8.2.b.i) del ER para los conflictos armados internacionales y el artículo 8.2.e.i) del ER para los conflictos sin carácter internacional-, modalidad que, como se mencionó, fue empleada por algunas unidades militares como parte de las campañas de DE en la ex-Yugoslavia.

Sin embargo, al igual que sucede con los crímenes de lesa humanidad (Cottier \& Baumgartner, 2016, p. 414), el crimen de guerra que ha quedado más asociado a la $\mathrm{DE}$ es el de la deportación o traslado de la población de un territorio ocupado, en el caso de un conflicto armado internacional - art. 8.2.b.viii) del ER- o el desplazamiento de la población civil por razones relacionadas con el conflicto, en caso de un conflicto armado sin carácter internacional - art. 8.2.e.viii) del ER- (Pégorier, 2013, p. 47). El primero de ellos ya era considerado una "infracción grave" en los términos del artículo 147 del IV Convenio de Ginebra - y del artículo 85.4.a) del Protocolo Adicional I-, dado que infringe la disposición del artículo 49 del mismo instrumento, en cuanto prohíbe los traslados y deportaciones de índole forzosa; mientras que el segundo constituye una violación a la prohibición de los desplazamientos forzados contenida en el artículo 17 del Protocolo Adicional II. Si bien en ningún caso se requiere la finalidad discriminatoria que caracteriza a la DE, se trata de uno de los medios más tristemente célebres de ejecución de esta y, como tal, basta para ser considerado como crimen de guerra.

En definitiva, es posible calificar como crímenes de guerra las distintas formas de comisión de actos de DE en la medida en que se satisfaga el elemento de contexto de estos crímenes, es decir, se cometan en un conflicto armado (tanto internacional como no internacional), lo cual ha sucedido en los casos modernos de DE.

\subsection{Conclusión: una política, muchos crímenes}

Como se advierte, la DE, pese a no ser un crimen como tal, es llevada a cabo mediante actos que sí son considerados crímenes en virtud del derecho internacional. Particularmente interesante es su vinculación con los crímenes de lesa humanidad, puesto que las condiciones de su comisión constituyen el elemento de contexto requerido para ellos, y las modalidades en que se ejecuta caen dentro de varios de los incisos del artículo $7^{\circ}$ del ER. No obstante, tampoco puede descartarse su carácter de crimen de guerra cuando es cometida durante un conflicto armado ni la posibilidad, reconocida por la CIJ, de que en algún caso pueda constituir genocidio. De esta manera, no vemos posible que actos ejecutados como parte de una campaña de DE no caigan dentro de alguno de los más graves crímenes de trascendencia para la comunidad internacional en su conjunto. 
La DE, por lo tanto, no es un crimen de derecho internacional como tal, pero todas las conductas empleadas en su ejecución sí lo son; de allí que no exista necesidad de tipificarla por separado. Esta conclusión, trasladada al ámbito de la RDP, no solamente no soluciona, sino que complejiza la discusión al respecto, como veremos a continuación.

\section{La depuración étnica y la responsabilidad de proteger}

A grandes rasgos, podemos considerar que la RDP implica que los Estados tienen la responsabilidad de proteger a su población de graves atrocidades masivas (el genocidio, los crímenes de guerra, la DE y los crímenes de lesa humanidad), y que cuando no ejerzan esa responsabilidad (por incapacidad o por falta de voluntad), la comunidad internacional, a través de las NU, puede adoptar medidas para ayudar a proteger a esa población, incluso mediante el uso de la fuerza armada.

El concepto fue acuñado en 2001 por la Comisión Internacional sobre Intervención y Soberanía de los Estados (CIISE), que incluyó la DE dentro de uno de los requisitos que justificarían una intervención militar en ejercicio de la RDP, al que denominó "causa justa”:

La intervención militar con fines de protección humana está justificada cuando se dan dos amplios conjuntos de circunstancias, es decir, cuando se trata de atajar o evitar:

- grandes pérdidas de vidas humanas, reales o previsibles, con o sin intención genocida, que sean consecuencia de la acción deliberada de un Estado, o de su negligencia o incapacidad de actuar o del colapso de un Estado; o

- una "depuración étnica" en gran escala, real o previsible, llevada a cabo mediante el asesinato, la expulsión forzosa, el terror o la violación (CIISE, 2001, pp. 35-36, énfasis añadido).

La CIISE aclara qué tipo de situaciones están incluidas dentro de estas dos condiciones, entre las que se encuentran:

- diferentes tipos de "depuración étnica", como el asesinato sistemático de los miembros de un grupo concreto con el fin de reducir o eliminar su presencia en una zona determinada; el traslado sistemático de los miembros de un grupo concreto fuera de una determinada zona geográfica; los actos de terror encaminados a 
forzar la huida de ciertas personas; y la violación sistemática de mujeres de un grupo determinado con objetivos políticos (ya sea como otra forma de terrorismo o para modificar la composición étnica de dicho grupo);

- los crímenes de lesa humanidad y las infracciones de las leyes de la guerra $[\ldots]$ que conlleven matanzas o depuraciones étnicas en gran escala (CIISE, 2001, p. 36).

Lo que caracteriza a las dos condiciones, resaltó la CIISE, es que los actos deben cometerse "a gran escala" para justificar una intervención. Aunque parece que la CIISE distingue entre graves pérdidas de vidas y $\mathrm{DE}$, ello no significa que esta última no implique pérdidas de vidas, sino que el hecho de que se lleve a cabo mediante expulsiones forzadas o violaciones sistemáticas basta para considerarla dentro del umbral que justifica la intervención, siempre que se cumpla con el requisito de escala (Kolb, 2003, pp. 130-131).

La RDP fue luego adoptada en 2004 en el informe del Grupo de Alto Nivel sobre las Amenazas, las Oportunidades y el Cambio (GAN), creado por el sG:

Aprobamos la norma que se está imponiendo en el sentido de que existe una responsabilidad internacional colectiva de proteger, que el Consejo de Seguridad puede ejercer autorizando la intervención militar como último recurso en caso de genocidio y otras matanzas en gran escala, de depuración étnica o de graves infracciones del derecho internacional humanitario que un gobierno soberano no haya podido o no haya querido prevenir (GAN, 2004, p. 63).

Bellamy (2010) afirma que el informe del GAN amplió sutilmente el umbral de justa causa de la CIISE, al agregar "graves infracciones del derecho internacional humanitario" a la lista de genocidio, matanzas en gran escala y DE. No obstante, la CIISE también se había referido a las violaciones del derecho humanitario en la medida en que conllevaran matanzas o DE a gran escala. Lo que sí advertimos aquí es la adopción de un criterio mixto fáctico-jurídico a la hora de definir el umbral de protección: junto a las matanzas y la DE, que son descripciones de situaciones de hecho (como lo había realizado la CIISE), el GAN agrega el genocidio y las graves violaciones del derecho humanitario, que son conceptos jurídicos. 
El SG también adoptó la RDP en el informe que presentó a la Cumbre Mundial 2005, en el que instó a los Estados miembros a que pidieran al cs que reafirmara el derecho de este "a utilizar la fuerza militar, incluso de forma preventiva, para preservar la paz y la seguridad internacionales, especialmente en casos de genocidio, depuración étnica y otros crímenes de lesa humanidad [...]” (Annan, 2005, p. 64); y a que adopten "el principio de la 'responsabilidad de proteger' como base para la acción colectiva contra el genocidio, la depuración étnica y los crímenes de lesa humanidad [...]" (Annan, 2005, p. 65).

Con respecto al umbral de protección, el SG también se aparta de las soluciones de la CIISE y del GAN, limitando la RDP al genocidio, la DE y 'otros' crímenes de lesa humanidad, inclinándose así por un criterio eminentemente jurídico - en el que no se mencionan las violaciones al derecho humanitario-, pero manteniendo a la DE como categoría de hecho separada, a la que parece considerar como un crimen de lesa humanidad.

Ya en el proceso de la Cumbre Mundial 2005, durante todas las etapas de redacción del proyecto de DF se mantuvo el criterio que había propuesto el sG, pero agregando los crímenes de guerra (que estaban en el informe del GAN como "graves infracciones del derecho internacional humanitario"). Jean Ping — presidente de la AG, a cargo de las negociaciones del DF- atribuyó esta fórmula al embajador de Pakistán, que se mantendría hasta el final en el proyecto (Luck, 2009, p. 13).

Así, la redacción definitiva del DF, aprobado por la Resolución 60/1 de la AG, incluyó cuatro menciones a la DE, siempre con referencia a la responsabilidad de proteger a las poblaciones "del genocidio, los crímenes de guerra, la depuración étnica y los crímenes de lesa humanidad".

Por lo que respecta al ámbito material de la RDP, las diferencias entre la CIISE $\mathrm{y}$ el DF son evidentes. La primera recurría a un criterio fáctico, es decir, descriptivo de una situación de hecho, mientras que el segundo parece inclinarse por un criterio jurídico, toda vez que tanto el genocidio como los crímenes de guerra y los crímenes de lesa humanidad son figuras típicas del derecho penal internacional. Ello podría plantear un importante inconveniente a la hora de determinar cuándo se han cometido o se están cometiendo tales crímenes, dado que, por ser figuras penales su determinación correspondería a tribunales, se debería esperar al menos la iniciación - cuando no la finalización - de un proceso penal internacional (Añaños Meza, 2010, p. 213), lo cual es un absurdo tratándose de la adopción de 
una respuesta 'oportuna y decisiva' en virtud de la RDP. En ese caso, la descripción de la CIISE, que requiere una mera constatación de hechos sin ninguna calificación jurídica, ${ }^{9}$ parecía más apropiada, ${ }^{10}$ aunque tal vez más vaga y susceptible de abuso.

El mayor problema para nuestro análisis, relacionado con el anterior, lo plantea la inclusión de la DE junto a los tres crímenes; a diferencia de estos, como vimos, la DE no constituye un tipo penal diferente y, según las circunstancias, podrá ser un crimen de lesa humanidad, un crimen de guerra o, incluso, un caso de genocidio, ${ }^{11}$ es decir, ya está incluida dentro de tales crímenes. ¿Por qué, entonces, se la agregó en el DF con categorías penales claramente definidas en el derecho internacional?

Strauss (2009) comparte con la mayoría de la doctrina que el término "depuración étnica" no tiene significación jurídica propia y que puede subsumirse en los tres crímenes, pero agrega que su inclusión entre los crímenes listados permite una evaluación de los eventos en el terreno más fáctica que jurídica, que debería ser explorada más sistemáticamente por su capacidad de proveer un punto de entrada para la aplicación de la RDP (pp. 315-316). Este último comentario lo indica como uno de los pocos autores que ha reparado en la diferencia de la DE con los crímenes como una característica con utilidad práctica para la evaluación de situaciones sin tener que caer en debates jurídicos respecto de las figuras penales.

Kleffner (2012) parte de la misma premisa para llegar a una conclusión diametralmente opuesta, al sostener que el concepto de RDP — como se lo entiende actualmente- mezcla estrictas nociones jurídicas en la forma de bien reconocidos crímenes en virtud del derecho internacional, con la noción más coloquial de DE. Tal inconsistencia conceptual vuelve a la mención separada de la DE redundante y puede reintroducir un elemento de vaguedad que la introducción de los crímenes internacionales pretendió reducir en primer lugar. En última instancia, destaca, esto podría socavar la utilidad operacional del concepto de RDP (Kleffner, 2012, p. 87).

9 Davis (2011) indica que la CIISE no define el umbral de la RDP a través del lenguaje del derecho penal internacional y el derecho de los derechos humanos, y que evita términos como genocidio o crímenes de lesa humanidad - aunque la cIISE entiende estos umbrales como inclusivos de muchos de tales crímenesen favor de unas directrices descriptivas más discrecionales, en marcado contraste con los informes y resoluciones de las $\mathrm{NU}$, que nombran los crímenes específicos que activan la RDP (p. 893). No estamos convencidos de que las directrices de la cIISE sean 'más discrecionales'. Ambas situaciones deben superar un umbral de maģnitud - 'a ģran escala'-, y, de hecho, hay ciertos crímenes que podrían no superar ese umbral, haciendo a la redacción del DF más amplia.

10 En contra, otros autores resaltan las virtudes de la clarificación al recurrir a categorías con un signnificado jurídico preciso (Bellamy, 2008, p. 623; Arredondo, Rodríguez \& Serbin, 2011, p. 10).

11 Tanto la falta de autonomía jurídica de la DE como la posibilidad de considerarla dentro de los otros crímenes es destacado por numerosos autores que discuten la RDP (Evans, 2008, pp. 12-13; Luck, 2008, p. 4; Arredondo, 2009, p. 252; Rosenberģ, 2009, p. 461; Brunée \& Toope, 2010, p. 195; Seyle \& Aloyo, 2015, pp. 184-185; Bellamy \& Reike, 2010, p. 278; Hilpold, 2015, p. 15), pero ninguno de ellos problematiza su inclusión en el DF. 
Por su parte, Scheffer (2008), sin separarse de la opinión mayoritaria, agrega que la DE es un término muy poderoso, tanto que existe una creciente base para considerarlo como un crimen de lesa humanidad que merece su propia designación en virtud de la lista de esos crímenes (pp. 128-129). El 'poder' del concepto, que, como vimos, parece provenir del lenguaje periodístico, parece cumplir un importante rol tanto en la selección original por la CIISE como por su permanencia en el DF. Como asevera Zyberi (2013), la inclusión separada junto con los crímenes tiene más connotaciones políticas que un significado jurídico real (p. 513).

Así, la inclusión de la DE en el ámbito material de la RDP en el DF probablemente obedezca a que el concepto se mantuvo desde la formulación original de la CIISE por su impacto en el lenguaje político, y nunca se planteó seriamente su eliminación, aunque en la actualidad coexista de manera anómala como única categoría fáctica junto a tres tipos jurídico-penales. ${ }^{12}$

\section{Consideraciones finales}

La DE es una atroz práctica seguida para la ejecución de una política discriminatoria, pero no un crimen internacional en sí misma: no es tipificada en ningún instrumento internacional ni su valor jurídico reconocido en la jurisprudencia internacional. Eso no implica sostener su licitud, puesto que, como hemos visto, puede constituir un acto de genocidio, un crimen de lesa humanidad o un crimen de guerra, dependiendo de las circunstancias. Es posible, entonces, afirmar que no constituye una categoría jurídica autónoma en el derecho internacional.

Así las cosas, resulta errónea su inclusión junto a las tres categorías de crímenes internacionales — por lo demás, muy bien establecidas en el ordenamiento jurídico- en el DF. En primer lugar, porque no comparte su misma naturaleza; y, en segundo, porque ya está comprendida dentro de ellos, como se demostró en las páginas anteriores.

Debemos enfatizar que la CIISE optó por recurrir a descripciones fácticas, mientras que en las NU se eligió usar categorías jurídicas, pero sin suprimir la DE. Esa, pensamos, es la causa de esta confusión, que en su formulación original no existía, lo cual dio lugar a una anomalía en el ámbito de alcance material de la RDP.

12 La presencia anómala de la DE junto a los crímenes ha traído un problema de lenguaje, ya que, al abordar el alcance material de la RDP, no es posible referirse a 'los cuatro crímenes', por lo que han debido elaborarse expresiones tales como "crímenes y actos" o "crímenes y violaciones", que suscitaron en sí mismas otras discusiones de parte de algunos Estados críticos del concepto, que temen una posible ampliación del ámbito de la RDP, cuando en realidad se trata de conceptos 'paraguas' que puedan reparar la anomalía de incluir a la DE junto con los crímenes (Bellamy, 2013; Pezzano, 2016). 
Es curioso que, ya en 1994, cuando la idea de RDP aún no existía, Petrovic (1994) alertaba:

It is essential that the new term is not used to replace pre-existing definitions in international law. So far the international community has been employing precisely this term, but only as an excuse not to comply with duties laid down by international law. The objective of this article has been to provide elements for future analysis of this phenomenon, as individual responsibility will be determined by the International Tribunal established for that purpose. In doing so it is hoped that the Tribunal will apply well defined tenants of international law rather than emotive phrases and terms, so far, 'ethnic cleansing' has been used merely as a political rather than as a legal term (p. 359).

Es significativo cómo el TPIY siguió este consejo, en cuanto no recurrió al término más que para describir situaciones de hecho, pero nunca para calificaciones jurídicas.

Sin embargo, no parece suceder lo mismo con la RDP, en cuyo ámbito material la DE fue incluida junto a nociones jurídicas concretas, generando un híbrido de difícil lectura. ¿Cuál es, entonces, su sentido? Pensamos que se abren varias opciones para el análisis. La primera implica afirmar que, por no tener significado jurídico independiente, no tiene sentido y fue un error mantenerla en la transición del método empleado por la CIISE al seguido en las NU. La segunda, abrevando en el sentido original empleado por la CIISE, en cuanto descripción fáctica, considera que permite un mayor grado de flexibilidad a la hora de determinar si se está cometiendo o no una atrocidad de estas características, ya que no estaría sujeta a los estrictos criterios jurídico-penales de los crímenes. Pero esta opción puede ser vista tanto positiva como negativamente, tal cual lo ilustran las posiciones doctrinarias encontradas al respecto.

Por nuestra parte, nos inclinamos por una tercera opción, relacionada con el impacto de la noción de $\mathrm{DE}$ en el lenguaje político internacional y a la vez con la crítica que se le efectuara de ser un eufemismo de genocidio. Esta crítica se basa, como dijimos, en que se pretende con ella eludir las obligaciones derivadas del derecho internacional (Pégorier, 2013). Pero, al incluir a la DE dentro del ámbito material de la RDP, queda cubierta - fuera de toda duda - por las obligaciones de los Estados de proteger a su población y también por la - aún discutida - 'responsabilidad' de la comunidad internacional de 'ayudar a proteger' a las poblaciones de la comisión de crímenes internacionales. 
De esa forma, la RDP — si es que continúa desarrollándose- puede ayudar a reducir el uso eufemístico de la noción de DE, por cuanto la calificación de una situación como tal no permitiría eludir las obligaciones de los Estados de proteger a la población, y así se daría un paso más en la protección de los seres humanos contra atrocidades semejantes.

\section{Referencias}

Ambos, K. (2011). Nociones básicas de derecho internacional humanitario. Valencia: Tirant lo Blanch.

Andreopoulos, G. J. (2004). Ethnic cleansing. Retrieved from https://www.britannica.com/topic/ethnic-cleansing

Annan, K. A. (2005). Un concepto más amplio de la libertad: desarrollo, seguridad y derechos humanos para todos. Informe del Secretario General, Documento A/59/2005. Recuperado de https://undocs.org/A/59/2005

Añaños Meza, M. C. (2010). La 'responsabilidad de proteger' en Naciones Unidas y la doctrina de la 'responsabilidad de proteger'. Anuario Mexicano de Derecho Internacional, $X, 199-244$.

Arredondo, R. (2009). La prevención del genocidio y la responsabilidad de proteger. En J. A. Consigli (Coord.), El genocidio ante la Corte Internacional de Justicia (pp. 231-267). Buenos Aires: CARI.

Arredondo, R., Rodríguez, G. M., \& Serbin, A. (2011). Responsabilidad de proteger y prevención en América Latina y el Caribe: el rol de la sociedad civil. Buenos Aires: CRIES.

Asamblea General de las Naciones Unidas. (1948a). Genocide - Draft Convention (E/794) and report of the Economic and Social Council. Syria: Amendment to Article II, Document A/C.6/234.

Asamblea General de las Naciones Unidas. (1948b). Sixth Committee - Eighty-Second Meeting. Document A/C.6/SR.82.

Bellamy, A. J. (2008). The responsibility to protect and the problem of military intervention. International Affairs, 84(4), 615-639.

Bellamy, A. J. (2010). The responsibility to protect. Five years on. Ethics \& International Affairs, 24(2), 143-169. 
Bellamy, A. J. (2013). Making RtoP a living reality: reflections on the 2012 General Assembly dialogue on timely and decisive response. Global Responsibility to Protect, 5, 109-125.

Bellamy, A. J., \& Reike, R. (2010). The responsibility to protect and international law. Global Responsibility to Protect, 2, 267-286.

Blum, R., Stanton, G. H., Sagi, S., \& Richter, E. D. (2008). 'Ethnic cleansing' bleaches the atrocities of genocide. European Journal of Public Health, 18(2), 204-209.

Brunée, J., \& Toope, S. J. (2010). The responsibility to protect and the use of force: building legality? Global Responsibility to Protect, 2, 191-212.

Bulutgil, H. Z. (2016). The roots of ethnic cleansing in Europe. New York: Cambridge University Press.

Cambridge Dictionary. (s. f.). Meaning of “cleanse". Retrieved from https://dictionary.cambridge.org/dictionary/english/cleanse

Comisión de Derecho Internacional de las Naciones Unidas. (2002). Anuario de la Comisión de Derecho Internacional 1996 (Vol. II, segunda parte). Recuperado de https://legal.un.org/ilc/publications/yearbooks/spanish/ilc_1996_v2_ p2.pdf

Comité para la Eliminación de la Discriminación Racial de las Naciones Unidas. (1996). Informe del Comité para la Eliminación de la Discriminación Racial. Documento A/50/18.

Comisión Internacional sobre Intervención y Soberanía de los Estados. (2001). La responsabilidad de proteger. Informe de la Comisión Internacional sobre Intervención y Soberanía de los Estados.

Comisión de Derechos Humanos de las Naciones Unidas. (1992). Informe sobre la situación de los derechos humanos en el territorio de la antigua Yugoslavia presentado por el Sr. Tadeusz Mazowiecki, relator especial de la Comisión de Derechos Humanos. Documento E/CN.4/1992/S-1/9. Recuperado de https:// digitallibrary.un.org/record/149074\#record-files-collapse-header

Conferencia Mundial de Derechos Humanos. (1993). Declaración y Programa de Acción de Viena. Documento A/CONF.157/23. Recuperado de https://undocs. org/es/A/CONF.157/23 
Consejo de Seguridad de las Naciones Unidas. (1993a). Informe presentado por el Secretario General de conformidad con el párrafo 2 de la Resolución 808 (1993) del Consejo de Seguridad. Documento S/25704. Recuperado de https://undocs.org/sp/S/25704

Consejo de Seguridad de las Naciones Unidas. (1993b). Informe provisional de la Comisión de Expertos establecida en la Resolución 780 (1992) del Consejo de Seguridad. Documento S/25274. Recuperado de https://undocs.org/S/25274

Consejo de Seguridad de las Naciones Unidas. (1994). Informe final de la Comisión de Expertos establecida en virtud de la Resolución 780 del Consejo de Seguridad (1992). Documento S/1994/674. Recuperado de https://undocs. org/S/1994/674

Cottier, M., \& Baumgartner, E. (2016). Article 8 paragraph 2(b)(viii): prohibited deportations and transfers in occupied. In K. Ambos \& O. Triffterer (Eds.), The Rome Statute of the International Criminal Court: a commentary (pp. 404416). C. H. Beck.

Cottier, M., \& Mzee, S. (2016). Article 8 paragraph 2(b)(xxii): rape and other forms of sexual violence. In K. Ambos \& O. Triffterer (Eds.), The Rome Statute of the International Criminal Court: a commentary (pp. 476-503). C. H. Beck.

Davis, T. (2011). Taking international law at its word and its spirit: re-envisioning responsibility to protect as a binding principle of international law. Florida State University Law Review, 38, 883-910.

Evans, G. (2008). The responsibility to protect. Ending mass atrocities crimes once and for all. Washington: Brookings Institution.

GAN. (2004). Un mundo más seguro: la responsabilidad que compartimos. Informe del Grupo de Alto Nivel sobre las Amenazas, los Desafíos y el Cambio. Documento A/59/565. Recuperado de https://undocs.org/A/59/565

Hilpold, P. (2015). From humanitarian intervention to the responsibility to protect. En P. Hilpold (Ed.), Responsibility to protect (R2P). A new paradigm of international law? (pp. 1-37). Leiden: Brill.

Kleffner, J. K. (2012). The scope of the crimes triggering the responsibility to protect. In J. Hoffmann \& A. Nollkaemper (Eds.), The responsibility to protect. From principle to practice (pp. 85-90). Amsterdam: Pallas Publications-Amsterdam University Press. 
Kolb, R. (2003). Note on humanitarian intervention. International Review of the Red Cross, 85(849), 119-134.

Luck, E. (2008). The United Nations and the responsibility to protect. The Stanley Foundation.

Luck, E. C. (2009). Sovereignty, choice, and the responsibility to protect. Global Responsibility to Protect, 1, 10-21.

Merriam-Webster Dictionary. (s. f.). Definition of "cleanse". Retrieved from https:// www.merriam-webster.com/dictionary/cleansing

Organización de las Naciones Unidas. (1992). La situación de los derechos humanos en el territorio de la antigua Yugoslavia. Nota del Secretario General. Documento A/47/635-S/24766. Recuperado de https://digitallibrary.un.org/ record $/ 153675 ? \ln =$ es

Pégorier, C. (2013). Ethnic cleansing. A legal qualification. Abingdon-New York: Routledge.

Petrovic, D. (1994). Ethnic cleansing. An attempt at methodology. European Journal of International Law, 5, 342-359.

Pezzano, L. (2016). La responsabilidad de proteger en las Naciones Unidas. Córdoba, España: CEA-UNC-Advocatus.

Quigley, J. B. (2006). The Genocide Convention. An international law analysis. Aldershot, Reino Unido: Ashgate.

Rosenberg, S. P. (2009). Responsibility to protect: a framework for prevention. Global Responsibility to Protect, 1, 442-477.

Schabas, W. (2007). Genocide and the International Court of Justice: finally, a duty to prevent the crime of crimes. Genocide Studies and Prevention: An International Journal, 2(2), 101-122.

Schabas, W. (2016). Article 6. In K. Ambos \& O. Triffeterer (Eds.), The Rome Statute of the International Criminal Court: a commentary (pp. 127-143). C. H. Beck.

Scheffer, D. (2008). Atrocity crimes framing the responsibility to protect. Western Reserve Journal of International Law, 40(1/2), 111-135.

Seyle, C., \& Aloyo, E. (2015). The role of business in the responsibility to protect. In D. Fiott \& J. Koops (Eds.), The responsibility to protect and the third pillar. Legitimacy and operationalization (pp. 171-189). Palgrave MacMillan. 
Strauss, E. (2009). A bird in the hand is worth two in the bush. On the assumed legal nature of the responsibility to protect. Global Responsibility to Protect, 1, 291-323.

Zyberi, G. (2013). Sharing the responsibility to protect: taking stock and moving forward. In G. Zyberi (Ed.), An institutional approach to the responsibility to protect (pp. 511-560). New York: Cambridge University Press.

\section{Jurisprudencia}

CIJ. Case concerning Application of the Convention on the Prevention and Punishment of the Crime of Genocide (Bosnia and Herzegovina v. Serbia and Montenegro), judgment, 26 de febrero de 2007. Citado como 'Genocidio'.

TPIY, Sala de Primera Instancia I. (1995). Prosecutor v. Dragan Nikolić ("Sušica Camp"). Caso No IT-94-2-R61, review of the indictment pursuant to rule 61 of the rules of procedure and evidence, 20 de octubre de 1995.

TPIY, Sala de Primera Instancia I. (1996). Prosecutor v. Radovan Karadžić and Ratko Mladć. Caso No IT-95-5-R61/IT-95-18-R61, review of the indictments pursuant to rule 61 of the rule of procedures and evidences, 11 de julio de 1996.

TPIY, Sala de Primera Instancia. (1997). Prosecutor v. Duško Tadić aka "Dule”. Caso No IT-94-1-T, sentencing judgment, 7 de mayo de 1997.

TPIY, Sala de Primera Instancia. (2000). Prosecutor v. Zoran Kupreškić, Mirjan Kupreškić, Vlatko Kupreškić, Drago Josipović, Dragan Papić, Vladimir Šantić. Caso No IT-95-16-T, judgement, 14 de enero de 2000.

TPIY, Sala de Primera Instancia. (2001). Prosecutor v. Radislav Krstić. Caso No IT-9833-T, judgement, 2 de agosto de 2001.

TPIY, Sala de Primera Instancia II. (2003). Prosecutor v. Milomir Stakić. Caso No IT-97-24-T, judgement, 31 de julio de 2003.

TPIY, Sala de Apelaciones. (2004). Prosecutor v. Dario Kordić and Mario Čerkez. Caso No IT-95-14/2, judgement, 17 de diciembre de 2004.

TPIY, Sala de Primera Instancia I. (2006). Prosecutor v. Momčilo Krajišnik. Caso No IT-00-39-T, judgement, 27 de septiembre de 2006. 